\title{
Branching Rules for Conformal Embeddings
}

\section{F. Levstein, J.I. Liberati}

Facultad de Matemática, Astronomía y Física, Universidad Nacional de Córdoba, Ciudad Universitaria, (5000) Córdoba, Argentina

Received: 25 June 1993 / in revised form: 24 November 1994

\begin{abstract}
We give explicit formulas for the branching rules of the conformal embeddings $s u(n(n+1) / 2)_{1} \supset s u(n)_{n+2}, \quad s u(n(n-1) / 2)_{1} \supset s u(n)_{n-2}, \quad s p(n)_{1} \supset s o(n)_{4} \oplus$ $s u(2)_{n}$, and $s o(m+n)_{1} \supset s o(m)_{1} \oplus s o(n)_{1}$ with $m$ and $n$ odd.
\end{abstract}

\section{Introduction}

The theory of affine Lie algebras has found very useful applications in Theoretical Physics. Our work is related to the models found in Conformal Field Theory.

In $[\mathrm{K}-\mathrm{P}]$ a set of functions called string functions were introduced to describe the branching rules of an integrable highest weight representation of an affine Lie algebra $\hat{g}$ with respect to its homogeneous Heisenberg subalgebra $\hat{h}$. There it was observed that those functions were modular functions with respect to a congruence subgroup of $S l_{2}(Z)$. In [K-W], the problem of describing the branching rules for an arbitrary pair $\hat{g} \supset \hat{p}$ was considered, proving modular properties and finding the asymptotic behaviour of most of them.

A special case of pairs $\hat{g} \supset \hat{p}$ comes from the so-called coset construction, [G-K-O], given an irreducible highest weight representation $L(\Lambda)$ of $\hat{g}$ one constructs the Sugawara operators $T_{m}^{\hat{g}}$ that give a representation of the Virasoro algebra on $L(\Lambda)$, similarly for the restriction to $\hat{p}$ one obtains a representation of the Virasoro algebra by operators $T_{m}^{\hat{p}}$. Taking the difference of the Virasoro operators, a new representation of the Virasoro algebra is obtained and it commutes with $\hat{p}$. Thus we get the decomposition:

$$
L(\Lambda)=\bigoplus_{\lambda} U(\Lambda, \lambda) \otimes L(\lambda)
$$



where

The central charge of the Virasoro algebra acting on $U(\Lambda, \lambda)$ is $z_{\Lambda}(g)-z_{\lambda}(p)$,

$$
z_{\Lambda}(g)=\frac{k \operatorname{dim} g}{k+h(g)}
$$

$h(g)$ being the dual Coxeter number, $k$ the level of $L(\Lambda)$ and $z_{\Lambda}(g)$ (resp. $\left.z_{\lambda}(p)\right)$ the central charge of the Sugawara representation of the Virasoro algebra acting on $L(\Lambda)$ (resp. $L(\lambda))$. Let $h$ and $\dot{h}$ denote Cartan subalgebras of $g$ and $p$. One can choose them so that $\dot{h} \subset h$. Let $H=\{\tau \in C \mid \operatorname{Im} \tau>0\}$ be the upperhalf plane. The normalized character $\chi_{\Lambda}$ of $L(\Lambda)$ is the holomorphic function on $H \times h$ :

$$
\chi_{\Lambda}(\tau, z)=q^{-z_{\Lambda}(g) / 24} \operatorname{tr}_{L(\Lambda)} \exp 2 i \pi\left(\tau L_{0}+z\right),
$$

where as usual $q$ denotes $\exp 2 i \pi \tau$. Suppose that $z \in \dot{h}$, then from $(0.1)$ we get:

$$
\chi_{\Lambda}(\tau, z)=\sum_{\lambda} b_{\lambda}^{\Lambda}(\tau) \chi_{\lambda}(\tau, z)
$$

where the branching function $b_{\lambda}^{\Lambda}$ is

$$
b_{\lambda}^{\Lambda}(\tau)=q^{-\left(z_{\Lambda}(g)-z_{\lambda}(p)\right) / 24} \operatorname{tr}_{U_{(\Lambda, \lambda)}} q^{L_{0}}
$$

The modular transformation properties of the characters are given by [KW]:

$$
\chi_{\Lambda}(\tau+1, z)=e^{2 \pi l\left(h_{\Lambda}-z_{\Lambda}(g) / 24\right)} \chi_{\Lambda}(\tau, z)
$$

with

$$
h_{\Lambda}=\frac{(\Lambda+2 \rho \mid \Lambda)}{2(k+h(g))} .
$$

$h_{\Lambda}$ is called the trace anomaly, and

$$
\chi_{\Lambda}\left(-\frac{1}{\tau}, \frac{z}{\tau}\right)=e^{i \pi(z \mid z) / \tau} \sum_{M \in P_{+}^{k}} a(\Lambda, M) \chi_{M}(\tau, z) .
$$

$P_{+}^{k}$ is the set of dominant highest weights of level $k$, and

$$
\begin{aligned}
a(\Lambda, M)= & i^{\left|\Delta_{+}\right|}\left|P / P^{*}\right|^{-1 / 2}(k+h(g))^{-n / 2} \\
& \times \sum_{w \in W} \operatorname{det}(w) \exp \frac{-2 i \pi}{k+h(g)}(\bar{\Lambda}+\bar{\rho} \mid w(\bar{M}+\bar{\rho})) .
\end{aligned}
$$

$\left|\Delta_{+}\right|$is the number of positive roots of $g, P$ is the weight lattice, $P^{*}$ its dual, $W$ the Weyl group, and $\bar{\Lambda}$ and $\bar{\rho}$ denote the "finite" parts of $\Lambda$ and $\rho$, i.e. $\Lambda=k \Lambda_{0}+\bar{\Lambda}, \rho=h(g) \Lambda_{0}+\bar{\rho}$, see $[\mathrm{K}]$.

Set $a(\Lambda)=a\left(\Lambda, k \Lambda_{0}\right)$. By the Weyl denominator formula,

$$
a(\Lambda)=\left|P / P^{*}\right|^{-1 / 2}(k+h(g))^{-n / 2} \prod_{\gamma \in \Delta_{+}} 2 \sin \frac{\pi(\bar{\Lambda}+\bar{\rho} \mid \alpha)}{k+h(g)} .
$$

Hence $a(\Lambda)$ is a positive real number, it is called the asymptotic dimension of $L(\Lambda)$, and appears in the asymptotic behavior of $\chi_{\Lambda}(\tau, 0)$ as $\tau \rightarrow 0$. It turns out 
to be:

$$
\chi_{\Lambda}(\tau, 0) \sim a(\Lambda) e^{l \pi z_{\Lambda}(g) / 12 \tau} .
$$

From (0.2) one easily deduces the transformation law of the branching function:

$$
b_{\lambda}^{\Lambda}\left(-\frac{1}{\tau}\right)=\sum_{M \in P_{+}^{k}, \mu \in \dot{P}_{+}^{k}} a(\Lambda, M) \dot{a}^{*}(\lambda, \mu) b_{\mu}^{M}(\tau)
$$

(dotted quantities refer to the subalgebra $p$ ).

We say that $g \supset p$ is a conformal embedding when $U(\Lambda, \lambda)$ is finite-dimensional, or equivalently when $z_{\Lambda}(g)=z_{\lambda}(p)$. This implies that the level of $\hat{g}$ is one. In this case $b_{\lambda}^{\Lambda}(\tau)=\operatorname{dim} U(\Lambda, \lambda)=b(\Lambda, \lambda)$ is a constant and $(0.10)$ reads:

$$
b(\Lambda, \lambda)=\sum_{M, \mu} a(\Lambda, M) \dot{a}^{*}(\lambda, \mu) b(M, \mu),
$$

i.e. the rectangular matrix $b(\Lambda, \lambda)$ commutes with the action of the modular group on the characters of $\hat{g}$ and $\hat{p}$. This matrix obeys also the important identity:

$$
a(\Lambda)=\sum_{\lambda \in \dot{P}_{+}^{k}} b(\Lambda, \lambda) \dot{a}(\lambda),
$$

obtained by inserting (0.9) and its analog for $p$ in $(0.2)$.

Conformal embeddings were classified in [B-B, S-W and A-G-O]. The problem of finding the branching rules for them was considered in [K-P, K-W, K-S, W, V and A-B-I]. We will give the branching rules for the families:

$$
\begin{array}{rll}
s u(n(n+1) / 2) \supset s u(n) & \text { index } & n+2, \\
s u(n(n-1) / 2) \supset \operatorname{su}(n) & \text { index } & n-2, \\
s p(n) \supset \operatorname{so}(n) \oplus \operatorname{su}(2) & \text { index } & (4, n), \\
s o(2(m+n+1)) \supset s o(2 m+1) \oplus \operatorname{so}(2 n+1) & \text { index } & (1,1) .
\end{array}
$$

The paper is organized as follows: we compute in the first four sections the decompositions corresponding to each of the cases mentioned above. Finally Sect. 5 contains some conclusions and remarks concerning modular invariant partition functions.

\section{Branching Rules for $s u(n) \subset s u(n(n+1) / 2)$}

The description of $b(\Lambda, \dot{\lambda})$ will be obtained from the study of the conformal pair

$$
u(1) \oplus \operatorname{su}(n) \subset \operatorname{so}(n(n+1)),
$$

which comes from the symmetric space

$$
S p(n) / U(n) .
$$

The link of (1.1) with

$$
s u(n)_{n+2} \subset s u(n(n+1) / 2)_{1}
$$


is provided by

$$
u(1) \oplus \operatorname{su}(n(n+1) / 2) \subset \operatorname{so}(n(n+1)),
$$

which is also conformal, with known branching rules (see below).

To compute the branching functions $b(\Lambda, \dot{\lambda})$ in the cases $(1.1)$ and $(1.2)$, we use the following theorem from [A-B-I], which is a generalization for the reductive case of the main theorem in [K-P] (with a correction by Nahm, see $[\mathrm{N}]$ ), and gives the decomposition of the half-spin representations $s$ and $t$. It is in fact a generalization of the finite-dimensional analog, which was proved in $[\mathrm{P}]$.

Theorem 1.1. Let $h$ be a simple Lie algebra, $p \subset h$ a reductive subalgebra of the same rank, such that $h=p \oplus V$ defines a symmetric space, i.e. $[V, V] \subset p$ and $p \subset g=s o(V)$. Then the decomposition of $s \oplus t$ of $\hat{g}$ into irreducible $\hat{p}$ modules is

$$
s \oplus t=\bigoplus_{w \in W / W} L(w(\rho)-\dot{\rho})
$$

where the dots refer to $p, W$ is the affine Weyl group of $h, \rho$ is the affine Weyl vector of $h$, and $W / \dot{W}$ is a set of coset representatives such that $w(\rho)-\dot{\rho}$ is a dominant weight of $\hat{p}$.

In the case (1.1) we have $h=s p(n), p=s u(n) \oplus u(1)$. The Weyl vector of $\hat{h}$ is given by $\rho=(n+1) \Lambda_{0}+\bar{\rho}$ with the (finite) Weyl vector $\bar{\rho}=n \varepsilon_{1}+$ $(n-1) \varepsilon_{2}+\cdots+\varepsilon_{n}$ (where the $\varepsilon_{l}$ are orthonormal vectors); in the case of $p$ we have $\dot{p}=n \dot{\Lambda}_{0}+\dot{\bar{\rho}}$ with $\dot{\bar{\rho}}=(n-1) \varepsilon_{1}+(n-2) \varepsilon_{2}+\cdots+\varepsilon_{n-1}-\frac{(n-1)}{2} \sum_{l=1}^{n} \varepsilon_{l}$ and the fundamental weights are $\dot{\Lambda}_{l}=\dot{\Lambda}_{0}+\dot{\bar{\Lambda}}_{l}$ with $\dot{\bar{\Lambda}}_{l}=\sum_{j=1}^{l} \varepsilon_{j}-\frac{i}{n} \sum_{j=1}^{n} \varepsilon_{j}$. The Weyl group is given by $W=\bar{W} n T$, where $T$ are the translations $\left\{t_{\alpha}\right\}_{\alpha \in L}$ with $L=$ $\sum_{i=1}^{n} 2 Z \varepsilon i=\sum_{i=1}^{n-1} 2 Z \alpha_{l}+Z \alpha_{n}\left(\left\{\alpha_{l}\right\}_{i=1}^{n}=\left\{\varepsilon_{1}-\varepsilon_{2}, \ldots, \varepsilon_{n-1}-\varepsilon_{n}, 2 \varepsilon_{n}\right\}\right.$ are the simple roots of $h)$, and $\bar{W}=S_{n} n\left(Z_{2}\right)^{n}$, where $S_{n}$ is the permutation group on the set $\left\{\varepsilon_{i}\right\}_{l=1}^{n}$ and $\left(Z_{2}\right)^{n}$ acts by $\varepsilon_{i} \rightarrow \pm \varepsilon_{i}$; in the case of $p, \dot{W}=S_{n} n \dot{T}$, where $T$ are the translations by $\alpha \in \dot{L}=\sum_{l=1}^{n-1} Z \dot{\alpha}_{l}$. Observe that $p$ is included in $h$ in such a way that $\dot{\alpha}_{k}=\alpha_{k}, 1 \leqq k \leqq n-1$, see $[\mathrm{K}]$.

In order to get a dominant weight $w(\rho)-\dot{\rho}$ we have to use as representatives of $T /(T \cap \dot{T})$ in $W / \dot{W}$, not the translations $t_{k \alpha_{n}}$ by multiples of $\alpha_{n}$, but the powers of $\sigma_{0} t_{\alpha_{n}}$, where $\sigma_{0}$ is the permutation

$$
1 \rightarrow 2 \rightarrow \cdots \rightarrow n \rightarrow 1
$$

since, if $\mu$ is the automorphism given by

$$
\mu\left(\dot{\Lambda}_{l}\right)=\dot{\Lambda}_{l+1} \bmod n
$$

then

$$
\sigma_{0} t_{\alpha_{n}}(w(\rho))-\dot{\rho}=\mu(w(\rho)-\dot{\rho}) .
$$

The restriction of $\lambda=\sum_{l=1}^{n} a_{i} \varepsilon_{l}$ a weight of $h$ to $p$ is $\dot{\lambda}=\sum_{l=1}^{n-1}\left(a_{l}-a_{l+1}\right) \dot{\bar{\Lambda}}_{l}$ and $\dot{\lambda}$ is a (strictly) dominant weight iff $a_{l}>a_{i+1}$. Thus we see that a suitable choice for $\bar{W} / \dot{\bar{W}}$ is the following: for each $s \in\left(Z_{2}\right)^{n}$ we take $\sigma_{s}$ the permutation which orders the coefficients of $s(\bar{\rho})=\sum_{i=1}^{n} a_{l} \varepsilon_{l}$ decreasingly. Then we take $\bar{W} / \dot{\bar{W}}=\left\{\sigma_{s} s\right\}$. 
For example, if $n=3$ and $s=(-1,1,1)$ then $s(\bar{\rho})=-3 \varepsilon_{1}+2 \varepsilon_{2}+1 \varepsilon_{3}$, so $\sigma_{s}=$ $1 \rightarrow 3 \rightarrow 2 \rightarrow 1$, and $\sigma_{s} s(\rho)-\dot{\rho}=2 \dot{\Lambda}_{0}+0 \dot{\Lambda}_{1}+3 \dot{\Lambda}_{2}$.

Therefore, we get the explicit form of the decomposition (1.3) in the case (1.1):

$$
s \oplus t=\bigoplus_{k \in Z} \bigoplus_{s \in\left(Z_{2}\right)^{n}} L\left(\mu^{k}\left(\sigma_{s} s(\rho)-\dot{\rho}\right)\right) \otimes F\left(h_{s, k}\right) .
$$

$F\left(h_{s, k}\right)$ is an irreducible Fock space representation of the $u(1)$ Heisenberg algebra with conformal weight $h_{s, k}$.

Recall that the decomposition (1.3) in the case (1.2) is (see [K-W]):

$$
s \oplus t=\bigoplus_{\Lambda \in Z} L\left(\Lambda \bmod \frac{n(n+1)}{2}\right) \otimes F\left(h_{\Lambda}\right),
$$

where $h_{\Lambda}=(\Lambda-n(n+1) / 4)^{2} /(n(n+1))$, and we identify the weights $\Lambda$ with the corresponding elements of $\frac{Z_{\frac{n(n+1)}{2}}}{\text {. }}$

In order to compare both decompositions we introduce some notations. For each $s=\left(s_{1}, \ldots, s_{n}\right) \in\left(Z_{2}\right)^{n},\left(s_{i}= \pm 1\right)$, we define

$$
c(s)=\sum_{i ; s_{i}=1}(n-i+1)
$$

i.e. $c(s)$ is the sum of the positive coefficients of $s(\bar{\rho})=\sum_{i=1}^{n} s_{l}(n-i+1) \varepsilon_{i}$; in the previous example $c(s)=3$.

We will need the following lemma:

Lemma 1.1. The trace anomaly of the weights in (1.6) is

$$
h_{\mu^{k}\left(\sigma_{s} s(\rho)-\dot{\rho}\right)}=\frac{(c(s)+k(n+1))(n(n+1)-2(c(s)+k(n+1)))}{2 n(n+1)} \bmod Z
$$

Proof. Since $h_{\dot{\lambda}}=\frac{(\dot{i}+2 \dot{\rho} \mid \dot{\lambda})}{4(n+1)}$ and $\mu^{k}\left(\sigma_{s} s(\rho)-\dot{\rho}\right)=\left(\mu^{k}\left(\sigma_{s} s(\rho)\right)-\dot{\rho}\right.$, it follows that

$$
h_{\mu^{k}\left(\sigma_{s} s(\rho)-\dot{\rho}\right)}=\frac{\left(\mu^{k}\left(\sigma_{s} s(\rho)\right)+\dot{\rho} \mid \mu^{k}\left(\sigma_{s} s(\rho)\right)-\dot{\rho}\right)}{4(n+1)}=\frac{\left|\mu^{k}\left(\sigma_{s} s(\rho)\right)\right|^{2}-(\dot{\rho} \mid \dot{\rho})}{4(n+1)}
$$

and it is easy to see that $(\dot{\rho} \mid \dot{\rho})=\frac{n\left(n^{2}-1\right)}{12}$. By definition we have $\sigma_{s} s(\rho)=$ $(n+1) \Lambda_{0}+\sum_{t=1}^{n} a_{i} \varepsilon_{i}$ (where $\left\{a_{i}\right\}_{l=1}^{n}=\left\{s_{i}(n-i+1)\right\}_{i=1}^{n}$ in decreasing order). Now we restrict $\sigma_{s} s(\rho)$ to the Cartan subalgebra of $s u(n)$,

$$
\begin{aligned}
\sigma_{s} s(\rho) & =2(n+1) \dot{\Lambda}_{0}+\sum_{i=1}^{n-1}\left(a_{l}-a_{i+1}\right) \dot{\bar{\Lambda}}_{i} \\
& =\left(2(n+1)+a_{n}+a_{1}\right) \dot{\Lambda}_{0}+\sum_{i=1}^{n-1}\left(a_{i}-a_{l+1}\right) \dot{\Lambda}_{l} .
\end{aligned}
$$


Thus we have

$$
\begin{aligned}
\mu^{k}\left(\sigma_{s} s(\rho)\right)= & \sum_{i=0}^{k-1}\left(a_{n-k+l}-a_{n-k+l+1}\right) \dot{\Lambda}_{i}+\left(2(n+1)+a_{n}+a_{1}\right) \dot{\Lambda}_{k} \\
& +\sum_{i=k+1}^{n-1}\left(a_{i-k}-a_{i-k+1}\right) \dot{\Lambda}_{i}
\end{aligned}
$$

and writing $\dot{\Lambda}_{i}$ as $\left[\varepsilon_{1}+\cdots+\varepsilon_{i}-\frac{i}{n}\left(\sum_{j=1}^{n} \varepsilon_{J}\right)\right]$ and taking $b=\sum_{l=1}^{n} a_{i}$, we obtain the following, modulo $\dot{\Lambda}_{0}$

$$
=\sum_{i=1}^{k} \frac{1}{n}\left(n a_{n-k+i}-b+2(n+1)(n-k)\right) \varepsilon_{i}+\sum_{i=k+1}^{n} \frac{1}{n}\left(n a_{i-k}-b-2(n+1) k\right) \varepsilon_{i} .
$$

Therefore $\left|\mu^{k}\left(\sigma_{s} s(\rho)\right)\right|^{2}=\frac{1}{n^{2}}\left\{\sum_{l=1}^{k}\left[4(n+1) n\left(n(n+1)+n a_{n-k+l}-b-2(n+1) k\right)\right]\right.$ $\left.+\sum_{i=1}^{n}\left(n a_{l}-b-2(n+1) k\right)^{2}\right\}=4(n+1)\left[k(n+1)+\sum_{l=1}^{k} a_{n-k+i}\right]+\frac{1}{n}[4(n+1) k$ $\left.(k(n-1)-b)+n \sum_{i=1}^{n} i^{2}-b^{2}\right]$, hence

$$
\begin{aligned}
h_{\mu^{k}\left(\sigma_{s} s(\rho)-\dot{\rho}\right)}= & k(n+1)+\sum_{l=1}^{k} a_{n-k+l}+\frac{1}{4 n(n+1)} \\
& \times\left(\frac{n^{2}(n+1)^{2}}{4}-b^{2}-4(n+1) k(b+k(1-n))\right),
\end{aligned}
$$

and since $c(s)=\frac{n(n+1)}{4}+\frac{b}{2}$ we prove the lemma.

Observe, that since $h_{s}+h_{t}=n(n+1) / 16$, with Lemma 1.1 we can determine $h_{s, k}$ in (1.6):

$$
h_{s, k}=(c(s)+k(n+1)-n(n+1) / 4)^{2} /(n(n+1)) \bmod Z .
$$

For each $s=\left(s_{1}, \ldots, s_{n}\right) \in\left(Z_{2}\right)^{n}$ and $\sigma \in S_{n}$, we denote by $\sigma(s)$ the action of $S_{n}$ on $\left(Z_{2}\right)^{n}$. Let $-s$ be given by $(-s)_{i}=-s_{i}$. The following lemma obtains the repetitions of the weights in (1.6), with an additional condition:

Lemma 1.2. Let $\sigma_{1}$ be the permutation defined by $\sigma_{1}(i)=n-i+1$. Then

a) $\sigma_{s} s(\rho)-\dot{\rho}=\mu^{k}\left(\sigma_{\sigma_{1}(-s)} \sigma_{1}(-s)(\rho)-\dot{\rho}\right)$ and $c(s) \equiv c\left(\sigma_{1}(-s)\right)+k(n+1)$ $\bmod \frac{n(n+1)}{2}$, with $k=\#\left\{i: s_{l}=1\right\}$.

b) If $\sigma_{s} s(\rho)-\dot{\rho}=\mu^{k}\left(\sigma_{\tilde{s}} \tilde{s}(\rho)-r\right)$, with $c(s) \equiv c(\tilde{s})+k(n+1) \bmod \frac{n(n+1)}{2}$, then $s=\tilde{s}, k=0$ or $\tilde{s}=\sigma_{1}(-s)$ with $k=\#\left\{i: s_{i}=1\right\}$.

Proof. a) Given $s \in\left(Z_{2}\right)^{n}$, let $a_{i}$ be as in the proof of Lemma 1.1, i.e. $\sigma_{s} s(\rho)=$ $(n+1) \Lambda_{0}+\sum_{l=1}^{n} a_{i} \varepsilon_{i}$ (where $\left\{a_{l}\right\}_{i=1}^{n}=\left\{s_{l}(n-i+1)\right\}_{i=1}^{n}$ in decreasing order). We define $A=\left\{i: s_{l}=1\right\}=\left\{i_{1}, \ldots, i_{k}\right\}$ with $i_{1}<\cdots<i_{k}$ and take the complement $A^{c}=\left\{i_{k+1}, \ldots, i_{n}\right\}$ with $i_{k+1}>\cdots>i_{n}$. Then

$$
a_{l}=\left\{\begin{array}{ll}
n-i_{l}+1 & l=1, \ldots, k \\
-\left(n-i_{l}+1\right) & l=k+1, \ldots, n
\end{array} .\right.
$$


We also have that $\hat{A}=\left\{n-i_{l}+1: l=k+1, \ldots, n\right\}$ and $\hat{A}^{c}=\left\{n-i_{l}+1: l=\right.$ $1, \ldots, k\}$ (where ^ refers to $\sigma_{1}(-s)$ ), and

$$
\begin{array}{rlrl}
\hat{a}_{l-k} & =i_{l} & l & =k+1, \ldots, n, \\
\hat{a}_{n-k+l} & =-i_{l} & l & =1, \ldots, k .
\end{array}
$$

Thus (a) follows from (1.8) and (1.9).

b) With the same notation as the previous lemmas, $c(s) \equiv c(\tilde{s})+k(n+1) \bmod$ $\frac{n(n+1)}{2}$ implies $\frac{b}{2} \equiv \frac{\tilde{b}}{2}+k(n+1) \bmod \frac{n(n+1)}{2}$. Then, using that $\mu^{k}\left(\sigma_{s} s(\rho)-\dot{\rho}\right)=$ $\mu^{k}\left(\sigma_{s} s(\rho)\right)-\dot{\rho}$.

$$
\sigma_{s} s(\rho)=2(n+1) \dot{\Lambda}_{0}+\sum_{i=1}^{n} \frac{1}{n}\left(n a_{i}-b\right) \varepsilon_{i}
$$

and from (1.10), we obtain that for some integer $j$

$$
a_{i}=\left\{\begin{array}{ll}
\tilde{a}_{n-k+i}+(j+2)(n+1) & i=1, \ldots, k \\
\tilde{a}_{l-k}+j(n+1) & i=k+1, \ldots, n
\end{array} .\right.
$$

Using that $\left\{a_{i}\right\}_{l=1}^{n}=\left\{s_{l}(n-i+1)\right\}_{l=1}^{n}$ in decreasing order, it is easy to see that $k$ must be the number of 1 's in $s$ and that

$$
j=\left\{\begin{array}{ll}
-1 & \text { if } k \neq 0 \\
0 \text { or } 1 & \text { if } k=0
\end{array} .\right.
$$

Thus the lemma follows from (1.13).

By Lemma 1.2.(b) it is natural to define the relation $s \sim \tilde{s}$ if $\tilde{s}=s$ or $\tilde{s}=\sigma_{1}(-s)$ and take $S$ a set of representatives in $\left(Z_{2}\right)^{n}$ for the quotient of $\left(Z_{2}\right)^{n}$ by this relation. Finally, we take $n_{s}=n$ if $s \neq \sigma_{1}(-s)$ and $n_{s}=n / 2$ otherwise. The next lemma gives the relation between the asymptotic dimensions.

Lemma 1.3. Let $\Lambda_{i}$ be the fundamental weights of $\operatorname{su}(n(n+1) / 2)$. Then

$$
\sum_{i=0}^{\frac{n(n+1)}{2}-1} a\left(\Lambda_{i}\right)=\sum_{s \in S} \sum_{k=0}^{n_{s}-1} a\left(\mu^{k}\left(\sigma_{s} s(\rho)-\dot{\rho}\right)\right) .
$$

Proof. Using the decompositions (1.6) and (1.7) and taking the character on both sides, we obtain

$$
\begin{aligned}
\sum_{\Lambda \in Z} \chi_{L\left(\Lambda \bmod \frac{n(n+1)}{2}\right)} \chi_{F\left(h_{\Lambda}\right)} & =\sum_{k \in Z} \sum_{s \in\left(Z_{2}\right) n} \chi_{\mu^{k}\left(\sigma_{s} s(\rho)-\dot{\rho}\right)} \chi_{F\left(h_{s, k}\right)}, \\
\sum_{k=0}^{\frac{n(n+1)}{2}-1} \sum_{j \in Z} \chi_{\Lambda_{k}} \chi_{F\left(h_{k+j n(n+1) / 2)}\right.} & =\sum_{k=0}^{n-1} \sum_{s \in\left(Z_{2}\right)^{n}} \sum_{j \in Z} \chi_{\mu^{k}\left(\sigma_{s} s(\rho)-\dot{\rho}\right)} \chi_{F\left(h_{s, k+j n}\right)}, \\
\sum_{k=0}^{\frac{n(n+1)}{2}-1} \chi_{\Lambda_{k}}\left(\sum_{j \in Z} \chi_{F\left(h_{k+m(n+1) / 2)}\right)}\right. & =\sum_{k=0}^{n-1} \sum_{s \in\left(Z_{2}\right)^{n}} \chi_{\mu^{k}\left(\sigma_{s} s(\rho)-\dot{\rho}\right)}\left(\sum_{j \in Z} \chi_{F\left(h_{s, k+m)}\right)}\right) .
\end{aligned}
$$


Recall that, in general,

$$
\chi_{F\left(h_{A}\right)}(\tau)=q^{h_{A}} / \eta(\tau)
$$

where as usual $q$ stands for $e^{2 \pi i \tau}$, with $\tau \in C, \operatorname{Im} \tau>0$, and $\eta$ is the Dedekind $\eta$-function.

Given $a, b \in \frac{1}{2} Z, a>0$, let (see [K, p. 259])

$$
f_{a, b}(\tau)=\sum_{j \in Z} q^{a(j+b / 2 a)^{2}}
$$

Then we obtain from (1.11) and (1.7),

$$
\sum_{J \in Z} \chi_{F\left(h_{k+j n(n+1) / 2}\right)}=f_{\frac{n(n+1)}{4}, k-\frac{n(n+1)}{4} / \eta}
$$

and

$$
\sum_{j \in Z} \chi_{F\left(h_{s, k+j n}\right)}=f_{n(n+1), \tilde{b}} / \eta
$$

with $\tilde{b}=2 c(s)+2 k(n+1)-n(n+1) / 2$.

Noting that, as $\tau \rightarrow 0$, we have

$$
\begin{aligned}
\eta(\tau)^{-1} & \sim(-i \tau)^{1 / 2} e^{\pi l / 12 \tau}, \\
f_{a, b}(\tau) & \sim(-i \tau)^{-1 / 2}(2 a)^{-1 / 2}, \\
\chi_{\Lambda}(\tau) & \sim a(\Lambda) e^{\pi i z_{\Lambda} / 12 \tau},
\end{aligned}
$$

we compute the asymptotic behavior of both sides of (1.15),

$$
\begin{aligned}
& \text { L.H.S. } \sim(n(n+1) / 2)^{-1 / 2} \sum_{k=0}^{\frac{n(n+1)}{2}-1} a\left(\Lambda_{k}\right) e^{\pi i z_{\Lambda_{k}} / 12 \tau+\pi i / 12 \tau}, \\
& \text { R.H.S. } \sim(2 n(n+1))^{-1 / 2} \sum_{k=0}^{n-1} \sum_{s \in\left(Z_{2}\right)^{n}} a\left(\mu^{k}\left(\sigma_{s} s(\rho)-\dot{\rho}\right)\right) e^{\pi i z} \mu^{k}\left(\sigma_{s} s(\rho)-\rho\right)^{/ 12 \tau+\pi l / 12 \tau} .
\end{aligned}
$$

Therefore we obtain the formula

$$
2 \sum_{i=0}^{\frac{n(n+1)}{2}-1} a\left(\Lambda_{i}\right)=\sum_{k=0}^{n-1} \sum_{s \in\left(Z_{2}\right)^{n}} a\left(\mu^{k}\left(\sigma_{s} s(\rho)-\dot{\rho}\right)\right) .
$$

In order to prove the lemma, we have to see that each one of the weights in the right-hand side of (1.14), appears twice in (1.16). First observe that, by Lemma 1.2.(a),

$$
\left\{\mu^{k}\left(\sigma_{s} s(\rho)-\dot{\rho}\right)\right\}_{k=0}^{n-1}=\left\{\mu^{k}\left(\sigma_{\sigma_{1}(-s)} \sigma_{1}(-s)(\rho)-\dot{\rho}\right)\right\}_{k=0}^{n-1} .
$$

Therefore, when $s \neq \sigma_{1}(-s), n_{s}=n$ and the asymptotic dimension of the weights in (1.17) are repeated in the right-hand side of (1.16). Finally, if $s=\sigma_{1}(-s)$ then $n$ must be even and (with the notation of Lemma 1.2) $k=\frac{n}{2}$, because $-s$ must have the same number of -1 's as $s$. Thus we have

$$
\sigma_{s} s(\rho)-\dot{\rho}=\mu^{\frac{n}{2}}\left(\sigma_{s} s(\rho)-\dot{\rho}\right) .
$$


So in the set (1.17) the weights $\mu^{k}\left(\sigma_{s} s(\rho)-\dot{\rho}\right), 0 \leqq k<\frac{n}{2}=n_{s}$ appear twice, completing the proof.

Now we are in a position to state our first basic result

Theorem 1.2. Let $\Lambda \in Z_{\frac{n(n+1)}{2}}$ denote a level one highest weight of $\operatorname{su}(n(n+1) / 2)$. Let $\dot{\lambda} \in \dot{P}_{+}^{(n+2)}$. Then the multiplicity $b(\Lambda, \dot{\lambda})$ of $L(\dot{\lambda})$ in $L(\Lambda)$ satisfies:

$$
\begin{array}{cl}
b(\Lambda, \dot{\lambda}) \neq 0 \quad \text { iff } \quad & \dot{\lambda}=\mu^{k}\left(\sigma_{s} s(\rho)-\dot{\rho}\right) \quad \text { for some } k \in Z, \quad s \in\left(Z_{2}\right)^{n} \\
& \text { and } \Lambda \equiv c(s)+k(n+1) \bmod \frac{n(n+1)}{2}
\end{array}
$$

and in this case $b(\Lambda, \dot{\lambda})=1$.

Proof. The center $Z_{n}$ of $s u(n)$ is embedded in the center $Z_{\frac{n(n+1)}{2}}$ of $\operatorname{su}(n(n+1) / 2)$ by the map $v \rightarrow v(n+1)$ in the additive notation. Since the elements of $Z_{n}$ and $Z_{\frac{n(n+1)}{2}}$ act as automorphisms of the corresponding algebras, this implies that

$$
b(\Lambda+v(n+1), v \dot{\lambda})=b(\Lambda, \dot{\lambda}) \text {. }
$$

Observe, that since $h_{\Lambda}=\frac{\Lambda(n(n+1)-2 \Lambda)}{2 n(n+1)}$, then

$$
h_{\Lambda}=h_{\Lambda^{\prime}} \bmod Z \quad \text { iff } \quad \Lambda^{\prime}=\Lambda \quad \text { or } \quad \Lambda^{\prime}=\frac{n(n+1)}{2}-\Lambda .
$$

If $b(\Lambda, \dot{\lambda}) \neq 0$, one derives (see, e.g., $[\mathrm{K}-\mathrm{W}]$ ) that

$$
h_{\Lambda}-h_{j} \in Z \text {, }
$$

and by the decompositions (1.6) and (1.7), $\dot{\lambda}=\mu^{k}\left(\sigma_{s} s(\rho)-\dot{\rho}\right)$ for some $k \in Z, s \in$ $\left(Z_{2}\right)^{n}$. We have to see that $\Lambda \equiv c(s)+k(n+1) \bmod \frac{n(n+1)}{2}$. We prove this in two steps. If $k=0$, (i.e. $\left.\dot{\lambda}=\sigma_{s} s(\rho)-\dot{\rho}\right)$ by Lemma $1.1,(1.19)$ and (1.20) we have

$$
\Lambda=c(s) \quad \text { or } \quad \Lambda=\frac{n(n+1)}{2}-c(s) .
$$

Observe that from (1.18) and (1.20) we get

$$
h_{\Lambda+(n+1)}-h_{\mu\left(\sigma_{s} s(\rho)-\rho\right)} \in Z
$$

then, due to Lemma 1.1 and (1.19) we have

$$
\Lambda+(n+1)=c(s)+(n+1) \quad \text { or } \quad \Lambda+(n+1)=\frac{n(n+1)}{2}-(c(s)+(n+1)),
$$

therefore $\Lambda=c(s)$. And the case $k \neq 0$ follows immediately from the previous case and (1.18).

Conversely, if $\dot{\lambda}=\mu^{k}\left(\sigma_{s} s(\rho)-\dot{\rho}\right)$ for some $k \in Z, s \in\left(Z_{2}\right)^{n}$, the decompositions (1.6) and (1.7) imply that there exist $\Lambda$ such that $b(\Lambda, \dot{\lambda}) \neq 0$, and by the previous considerations we have that $\Lambda$ must satisfy $\Lambda \equiv c(s)+k(n+1) \bmod \frac{n(n+1)}{2}$.

Finally, it follows from the asymptotic behavior (see Lemma 1.3), Lemma 1.2.(b) and (0.12), that $b(\Lambda, \dot{\lambda})=1$ if $b(\Lambda, \dot{\lambda}) \neq 0$, completing the proof. 
Example. To illustrate the use of the theorem, we will compute in the case $n=3$, i.e. the conformal pair

$$
\operatorname{su}(3)_{5} \subseteq s u(6),
$$

the decomposition of $L\left(\Lambda_{1}\right)$. By Theorem 1.2 we are interested in $s=\left(s_{1}, s_{2}, s_{3}\right) \in$ $\left(Z_{2}\right)^{3}$ and $0 \leqq k<3$ such that $1 \equiv c(s)+4 k \bmod (6)$, where $c(s)=\sum_{i: s_{i}=1}(4-$ $i)$. If $k=0$ then $c(s)=1$ and the only possibility is $s=(-1,-1,1)$; if $k=1$ then $c(s)=3$, and we have $s=(-1,1,1)$ or $s=(1,-1,-1)$. Finally, if $k=2$ then $c(s)=5$, and $s=(1,1,-1)$. But $(-1,-1,1) \sim(-1,1,1)$ and $(1,-1,-1) \sim$ $(1,1,-1)$. Therefore we must take $\sigma_{s} s(\rho)-\dot{\rho}(k=0)$ with $s=(-1,-1,1)$, and $\mu\left(\sigma_{s} s(\rho)-\dot{\rho}\right)(k=1)$ with $s=(1,-1,-1)$. So we obtain

$$
L\left(\Lambda_{1}\right)=L\left(3 \dot{\Lambda}_{0}+2 \dot{\Lambda}_{1}\right)+L\left(2 \dot{\Lambda}_{1}+3 \dot{\Lambda_{2}}\right) .
$$

\section{Branching Rules for $s u(n) \subset s u(n(n-1) / 2)$}

The idea is essentially the same as in Sect. 1 . Here the description of $b(\Lambda, \dot{\lambda})$ will be obtained from the study of the conformal pair

$$
u(1) \oplus \operatorname{su}(n) \subset \operatorname{so}(n(n-1))
$$

which comes from the symmetric space

$$
S O(2 n) / U(n) .
$$

And in this case the link of (2.1) with

$$
s u(n)_{n-2} \subset s u(n(n-1) / 2)_{1}
$$

is provided by

$$
u(1) \oplus \operatorname{su}(n(n-1) / 2) \subset \operatorname{so}(n(n-1)),
$$

which is also conformal.

We use Theorem 1.1 to obtain the decomposition of $s$ and $t$ in the case (2.1). We have $h=s o(2 n)$ and $p=s u(n) \oplus u(1)$. The fundamental weights of $h$ are $\bar{\Lambda}_{i}=$ $\sum_{j=1}^{l} \varepsilon_{j}(1 \leqq i \leqq n-2), \quad \bar{\Lambda}_{n-1}=\frac{1}{2}\left(\varepsilon_{1}+\cdots+\varepsilon_{n-1}-\varepsilon_{n}\right), \bar{\Lambda}_{n}=\frac{1}{2}\left(\varepsilon_{1}+\ldots+\varepsilon_{n}\right)$, and the Weyl vector is given by $\rho=(2 n-2) \Lambda_{0}+\bar{\rho}$ with the (finite) Weyl vector $\bar{\rho}=(n-1) \varepsilon_{1}+(n-2) \varepsilon_{2}+\cdots+\varepsilon_{n-1}$ (where the $\varepsilon_{i}$ are orthonormal vectors). The Weyl group is given by $W=\bar{W} n T$, where $T$ are the translations $\left\{t_{\alpha}\right\}_{\alpha \in L}$ with $L=$ $\sum_{i=1}^{n} Z \alpha_{i}\left(\left\{\alpha_{i}\right\}_{l=1}^{n}=\left\{\varepsilon_{1}-\varepsilon_{2}, \ldots, \varepsilon_{n-1}-\varepsilon_{n}, \varepsilon_{n-1}+\varepsilon_{n}\right\}\right)$, and $\bar{W}$ the semidirect product of the permutation group and $\left(Z_{2}\right)_{\text {even }}^{n}=\left\{\left(s_{1}, \ldots, s_{n}\right) \in\left(Z_{2}\right)^{n}: \#\left\{i: s_{l}=-1\right\}\right.$ is even $\}$. In the case of $p, \dot{\bar{\Lambda}}_{i}, \dot{\Lambda}_{i}, \dot{\bar{\rho}}, \dot{\rho}$ and $\dot{W}$ are as in Sect. 1 .

In order to get a dominant weight $w(\rho)-\dot{\rho}$ we have to use as representatives of $T / \dot{T}$ in $W / \dot{W}$, not the translations $t_{k \alpha_{n}}$ by multiples of $\alpha_{n}$, but the powers of $\tilde{\sigma}_{0} t_{\alpha_{n}}$, where $\tilde{\sigma}_{0}$ is the permutation $\sigma_{0}^{2}$ (see (1.4)), since, if $\mu$ is the automorphism given in Sect. 1, then

$$
\tilde{\sigma}_{0} t_{\alpha_{n}}(w(\rho))-\dot{\rho}=\mu^{2}(w(\rho)-\dot{\rho}) .
$$

As in Sect. 1, the restriction of $\lambda=\sum_{i=1}^{n} a_{i} \varepsilon_{i}$ a weight of $h$ to $p$ is $\dot{\lambda}=$ $\sum_{i=1}^{n-1}\left(a_{i}-a_{i+1}\right) \dot{\bar{\Lambda}}_{i}$ and $\dot{\lambda}$ is a (strictly) dominant weight iff $a_{i}>a_{i+1}$. Thus we 
see that a suitable choice for $\bar{W} / \dot{\bar{W}}$ is the following: for each $s \in\left(Z_{2}\right)_{\text {even }}^{n}$ we take $\sigma_{s}$ the permutation which orders the coefficients of $s(\bar{\rho})=\sum_{l=1}^{n} a_{i} \varepsilon_{i}$ decreasingly. For example, if $n=3$ and $s=(-1,1,-1)$ (even number of sign changes), then $s(\bar{\rho})=-2 \varepsilon_{1}+1 \varepsilon_{2}-0 \varepsilon_{3}$, so $\sigma_{s}=1 \rightarrow 3 \rightarrow 2 \rightarrow 1$.

Remark. 2.1. In this case the coefficients of the $\varepsilon_{i}$ in $\rho$ are different from those of Sect. 1 , and $\varepsilon_{n}$ has coefficient 0 . Since we are taking $s(\rho)$, then we can think that an $s \in\left(Z_{2}\right)^{n}$ with an even number of sign changes, belongs to $\left(Z_{2}\right)^{n-1}$ and acts in $\left\{\varepsilon_{1}, \ldots, \varepsilon_{n-1}\right\}$.

Therefore, we get the explicit form of the decomposition (1.3) in the case (2.1):

$$
s \oplus t=\bigoplus_{k \in Z} \bigoplus_{s \in\left(Z_{2}\right)_{\mathrm{even}}^{n}} L\left(\mu^{2 k}\left(\sigma_{s} s(\rho)-\dot{\rho}\right)\right) \otimes F\left(h_{s, k}\right) .
$$

As in Sect. 1, the decomposition (1.3) in the case (2.2) is:

$$
s \oplus t=\bigoplus_{\Lambda \in Z} L\left(\Lambda \bmod \frac{n(n-1)}{2}\right) \otimes F\left(h_{\Lambda}\right),
$$

where $h_{\Lambda}=(\Lambda-n(n-1) / 4)^{2} /(n(n-1))$, and we identify the weights $\Lambda$ with the corresponding elements of $Z_{\frac{n(n-1)}{2}}$.

In this case the numbers $c(s)$ are defined as follows. For each $s=\left(s_{1}, \ldots, s_{n}\right) \in$ $\left(Z_{2}\right)_{\text {even }}^{n},\left(s_{i}= \pm 1\right)$, we define

$$
c(s)=\sum_{i: s_{l}=1}(n-i),
$$

i.e. $c(s)$ is the sum of the positive coefficient of $s(\bar{\rho})=\sum_{i=1}^{n-1} s_{l}(n-i) \varepsilon_{l}$.

We will need the following result, which is similar to Lemma 1.1:

Lemma 2.1. The trace anomaly of the weights in (2.3) is

$$
h_{\left.\mu^{2 k}\left(\sigma_{s} s(\rho)-\rho\right)\right)}=\frac{(c(s)+2 k(n-1))(n(n-1)-2(c(s)+2 k(n-1)))}{2 n(n-1)} \bmod Z .
$$

Proof. See Lemma 1.1.

For each $s \in\left(Z_{2}\right)_{\text {even }}^{n}$, we define $\beta(s)=\sigma_{1}\left(-s_{1}, \ldots,-s_{n-1}, s_{n}\right)$. The following lemma obtains the repetitions of the weights in (2.3), with an additional condition:

Lemma 2.2. a) If $n$ is even and $0 \leqq k \leqq \frac{n}{2}$, then $\sigma_{s} s(\rho)-\dot{\rho}=\mu^{2 k}\left(\sigma_{\bar{s}} \tilde{s}(\rho)-\dot{\rho}\right)$, with $c(s) \equiv c(\tilde{s})+2 k(n-1) \bmod \frac{n(n-1)}{2}$ iff $s=\tilde{s} . k=0$ or $\tilde{s}=\sigma_{1}(-s)$ with $2 k=$ $\#\left\{i: s_{i}=1\right\}$.

b) If $n$ is odd and $0 \leqq k<n$, then $\sigma_{s} s(\rho)-\dot{\rho}=\mu^{2 k}\left(\sigma_{\bar{s}} \tilde{s}(\rho)-\dot{\rho}\right)$, with $c(s) \equiv$ $c(\tilde{s})+2 k(n-1) \bmod \frac{n(n-1)}{2}$ iff $\tilde{s}=\beta^{r}(s)$ with $r=0,1,2$ or 3 , and $2 k+s_{n}=\#\{i$ : $\left.s_{l}=1\right\}$ if $r=1$.

The proof is similar to that of Lemma 1.2. Now by this result, it is natural to define that $s \sim \tilde{s}$, in the case $n$ even, if $s=\tilde{s}$ or $\tilde{s}=\sigma_{1}(-s)$, and we take $n_{s}=n / 2$ if $\tilde{s} \neq \sigma_{1}(-s), n_{s}=n / 4$ otherwise; and in the case $n$ odd, $s \sim \tilde{s}$ if $\tilde{s}=\beta^{r}(s)$ with $r=0,1,2$ or 3 and we put $n_{s}=n$. Finally, we define $S$ a set of representatives 
in $\left(Z_{2}\right)_{\text {even }}^{n}$ for the quotient of $\left(Z_{2}\right)_{\text {even }}^{n}$ by this relation. The next lemma gives the relation between the asymptotic dimensions and the proof is basically the same as that of Lemma 1.3.

Lemma 2.3. Let $\Lambda_{l}$ be the fundamental weights of $s u(n(n-1) / 2)$. Then

$$
\sum_{l=0}^{\frac{n(n-1)}{2}-1} a\left(\Lambda_{l}\right)=\sum_{s \in S} \sum_{k=1}^{n_{s}} a\left(\mu^{2 k}\left(\sigma_{s} s(\rho)-\dot{\rho}\right)\right) .
$$

Now we are in a position to state our second basic result

Theorem 2.1. Let $\Lambda \in Z_{\frac{n(n-1)}{2}}$ denote a level one highest weight of $\operatorname{su}(n(n-1) / 2)$. Let $\dot{\lambda} \in \dot{P}_{+}^{(n-2)}$. Then the multiplicity $b(\Lambda, \dot{\lambda})$ of $L(\dot{\lambda})$ in $L(\Lambda)$ satisfies:

$$
\begin{gathered}
b(\Lambda, \dot{\lambda}) \neq 0 \quad \text { iff } \quad \dot{\lambda}=\mu^{2 k}\left(\sigma_{s} s(\rho)-\dot{\rho}\right) \quad \text { for some } k \in Z, s \in\left(Z_{2}\right)^{n-1} \\
\text { and } \Lambda \equiv c(s)+2 k(n-1) \bmod \frac{n(n-1)}{2}
\end{gathered}
$$

and in this case $b(\Lambda, \dot{\lambda})=1$.

Proof. See Theorem 1.2.

\section{The Branching Rules for $s p(n) \supset \operatorname{so}(n)_{4} \oplus s u(2)_{n}$}

We consider Cartan subalgebras $h, \dot{h}$ and $\ddot{h}$ of $\widehat{s p}(n), \widehat{s o}(n)$ and $\widehat{s u}(2)$ respectively, such that, $h \supset \dot{h} \oplus \ddot{h}$. We take a triangular decomposition $\widehat{s} p(n)=n_{-}+h+n_{+}$and in the same way $\dot{n}_{ \pm}$and $\ddot{n}_{ \pm}$, such that they are contained in $n_{ \pm}$. In this section, single dots refer to $s o(n)$ and double dots to $s u(2)$. With respect to these Cartan subalgebras we have the systems of simple roots. Let $\left\{\Lambda_{i}\right\}_{0}^{n},\left\{\dot{\Lambda}_{i}\right\}_{0}^{n / 2}$ and $\left\{\ddot{\Lambda}_{i}\right\}_{0}^{1}$ the respective dual root basis.

When $n=2 m$ let

$$
\begin{aligned}
\dot{\lambda}_{0} & =2 \dot{\Lambda}_{0} & & \dot{\lambda}_{0}^{\prime}=2 \dot{\Lambda}_{1} \\
\dot{\lambda}_{m} & =2 \dot{\Lambda}_{m} & & \dot{\lambda}_{m}^{\prime}=2 \dot{\Lambda}_{m-1} \\
\dot{\lambda}_{i} & =\dot{\Lambda}_{l-1}+\dot{\Lambda}_{i} & & i \in\{1, m-1\} \\
\dot{\lambda}_{l} & =\dot{\Lambda}_{l} & & 1<i<m-1 \\
\dot{\lambda}_{i} & =\dot{\lambda}_{n-l} & & 0<i<m .
\end{aligned}
$$

For $n=2 m+1$ let

$$
\begin{aligned}
& \dot{\lambda}_{0}=2 \dot{\Lambda}_{0} \quad \dot{\lambda}_{0}^{\prime}=2 \dot{\Lambda}_{1} \\
& \dot{\Lambda}_{1}=\dot{\Lambda}_{0}+\dot{\Lambda}_{1} \quad \dot{\lambda}_{m}=2 \dot{\Lambda}_{m} \\
& \dot{\lambda}_{i}=\dot{\Lambda}_{i} \quad 1<i<m-1 \\
& \dot{\lambda}_{i}=\dot{\lambda}_{n-l} \quad 1 \leqq i \leqq m .
\end{aligned}
$$


Now we can state our third main result:

Theorem 3.1. For the conformal embedding $\operatorname{sp}(n) \supset \operatorname{so}(n)_{4} \oplus s u(2)_{n}$ we have the following decompositions of the representations of level one of $\operatorname{sp}(n)$ :

a) If $n=2 m$ and $j \neq m$, then

$$
\begin{gathered}
L\left(\Lambda_{j}\right)=\sum_{j \leqq 2 i \leqq n+J} L\left(\dot{\lambda}_{i}+\dot{\lambda}_{|l-1|}\right) \otimes L\left((n-2 i+j) \ddot{\Lambda}_{0}+(2 i-j) \ddot{\Lambda}_{1}\right) \\
\oplus L\left(\dot{\lambda}_{J}+\dot{\lambda}_{0}^{\prime}\right) \oplus L\left((n-j) \ddot{\Lambda}_{0}+j \ddot{\Lambda}_{1}\right) \\
\oplus L\left(\dot{\lambda}_{m}^{\prime}+\dot{\lambda}_{|m-\jmath|}\right) \otimes L\left(j \ddot{\Lambda}_{0}+(n-j) \ddot{\Lambda}_{1}\right), \\
L\left(\Lambda_{m}\right)=\sum_{m \leqq 2 l \leqq n+m} L\left(\dot{\lambda}_{i}+\dot{\lambda}_{|m-i|}\right) \otimes L\left((n-2 i+m) \ddot{\Lambda}_{0}+(2 i-m) \ddot{\Lambda}_{1}\right) \\
\oplus L\left(\dot{\lambda}_{m}^{\prime}+\dot{\lambda}_{0}^{\prime}\right) \otimes L\left(m \ddot{\Lambda}_{0}+m \ddot{\Lambda}_{1}\right) .
\end{gathered}
$$

b) If $n=2 m+1$ and $j=2 k$ or $j=2 k-1$, then

$$
\begin{gathered}
L\left(\Lambda_{J}\right)=\sum_{i=k}^{m+k} L\left(\dot{\lambda}_{i}+\dot{\lambda}_{l-j \mid}\right) \otimes L\left((n-2 i+j) \ddot{\Lambda}_{0}+(2 i-j) \ddot{\Lambda}_{1}\right) \\
\oplus L\left(\dot{\lambda}_{J}+\dot{\lambda}_{0}^{\prime}\right) \otimes L\left((n-j) \ddot{\Lambda}_{0}+j \ddot{\Lambda}_{1}\right) .
\end{gathered}
$$

Remark. 3.1. If $n=2 m$, the sum is over the integers between $j / 2$ and $m+j / 2$, so we will have another term if $j$ is even. Observe that all the weights in the decomposition are different, so the multiplicities are one.

In order to prove the theorem, we will need the following lemma:

Lemma 3.1. The trace anomalies of the weights in (3.3) and (3.4) are the following:

a) $h_{\Lambda}=\frac{J(2 n+2-J)}{4(n+2)}$,

b) $h_{(n-j) \ddot{\Lambda}_{0}+j \ddot{\Lambda}_{1}}=\frac{j(j+2)}{4(n+2)}$,

c) $h_{\lambda_{1}+\lambda_{k}}=\frac{i(n-i)+k(n-k+2)}{2(n+2)}, \quad 0 \leqq k \leqq i \leqq m$.

Proof. Given $\Lambda \in P_{+}^{(m)}(g)$, the number $h_{\Lambda}$ can be calculated as follows. Let $\bar{\Lambda}=$ $\sum_{l=1}^{l} k_{l} \bar{\Lambda}_{i}$ and let $\left(\tilde{a}_{i j}\right)$ be the inverse of the Cartan matrix of $g$, then

$$
h_{\Lambda}=\sum_{l, j=1}^{l} \tilde{a}_{l j} k_{i}\left(k_{j}+2\right) / 2(m+h(g)) \text {. }
$$

Now, the lemma follows from this formula.

Proof of Theorem 3.1. First we show that the right-hand side of (3.3) and (3.4) is contained in $L\left(\Lambda_{j}\right)$. For this we use the decompositions (see $[\mathrm{K}-\mathrm{W}], \mathrm{p} .212$ )

$$
L\left(\Lambda_{j}\right)=\sum_{k=0}^{n-1} \sum_{s \in Z} \dot{L}\left(\dot{\Lambda}_{k}+\dot{\Lambda}_{k-j}\right) \otimes F(2 k-j-2 s n)
$$


of $\widehat{s p}(n) \supset \widehat{s u}(n)_{2} \times \hat{u}(1)$, in this case $\dot{\Lambda}_{k}$ are the fundamental weights of $\widehat{s u}(n)$. Also, we have $\widehat{s u}(n) \supset \widehat{s o}(n)_{2}$, and the restrictions of the fundamental weights of $\widehat{s u}(n)$ to $\widehat{s o}(n)$ are given by the $\dot{\lambda}_{l}$ in (3.1) and (3.2). From (3.5), there are weight vectors in $L\left(\Lambda_{j}\right)$ that are highest weight vectors in $L\left(\Lambda_{j}\right)$ as $\widehat{s u}(n)$-module, with weights $\dot{\Lambda}_{k}+\dot{\Lambda}_{k-j}$, with $0 \leqq k \leqq n-1$. And therefore, they are highest weight vectors in $L\left(\Lambda_{j}\right)$ as $\widehat{\operatorname{so}}(n)$-module, with weights $\dot{\lambda}_{i}+\dot{\lambda}_{|i-j|}$, with $j / 2 \leqq i \leqq(n+j) / 2$, which are all the different weights that appear in the restrictions. Since the action of $\widehat{s u}(2)$ commutes with the action of $\widehat{s o}(n)$, applying elements of $\ddot{n}_{+}$, we get a highest weight vector for $\widehat{s o}(n) \times \widehat{s u}(2)$, with weight $\dot{\lambda}_{l}+\dot{\lambda}_{|l-j|}$ and $(n-k) \ddot{\Lambda}_{0}+k \ddot{\Lambda}_{1}$ for some $k$. Now using Lemma 3.1, we see that the only possibility for $k$ that satisfies $(1.20)$ is $(2 i-j)$. Finally, using the automorphism that comes from the Dynkin diagram, we obtain the terms involving $\dot{\lambda}_{k}^{\prime}$ in (3.3) and (3.4).

In order to finish the proof we show that the asymptotic dimensions of both sides of (3.3) and (3.4) coincide. For this we make use of the formulas:

$$
\begin{aligned}
a\left((n-k) \ddot{\Lambda}_{0}+k \ddot{\Lambda}_{1}\right) & =\sqrt{\frac{2}{n+2}} \sin \frac{(k+1) \pi}{n+2}, \\
a\left(\Lambda_{j}\right) & =\sqrt{\frac{2}{n+2}} \sin \frac{(j+1) \pi}{n+2} .
\end{aligned}
$$

If $n=2 m \quad a\left(\dot{\lambda}_{l}+\dot{\lambda}_{j}\right)=\frac{4}{n+2} \sin \frac{(i+j+1) \pi}{n+2} \sin \frac{(j-i+1) \pi}{n+2}$

$$
\begin{gathered}
0<i \leqq j<m, \\
a\left(\dot{\lambda}_{l}+\dot{\lambda}_{J}\right)=\frac{2}{n+2} \sin ^{2} \frac{(i+j+1) \pi}{n+2} \quad i \in\{0, m\} \quad 0 \leqq j \leqq m .
\end{gathered}
$$

If $n=2 m+1 a\left(\dot{\lambda}_{i}+\dot{\lambda}_{j}\right)=\frac{4}{n+2} \sin \frac{(i+j+1) \pi}{n+2} \sin \frac{(j-i+1) \pi}{n+2}$

$$
0<i \leqq j<m,
$$

$$
a\left(\dot{\lambda}_{0}+\dot{\lambda}_{j}\right)=\frac{2}{n+2} \sin ^{2} \frac{(j+1) \pi}{n+2} \quad 0 \leqq j \leqq m
$$

which are proven by induction from the definition. Recall that $a(\Lambda)=a(\sigma \cdot \Lambda)$ for any automorphism $\sigma$ of the Dynkin diagram, then we obtain the asymptotic dimensions of the weights involving $\dot{\lambda}_{k}^{\prime}$.

So we must show, in the case $n=2 m+1$,

$$
\begin{aligned}
& \sum_{i=k}^{m+k} a\left(\dot{\lambda}_{l}+\dot{\lambda}_{|i-\jmath|}\right) a\left((n-2 i+j) \ddot{\Lambda}_{0}+(2 i-j) \ddot{\Lambda}_{1}\right)+a\left(\dot{\lambda}_{j}+\dot{\lambda}_{0}^{\prime}\right) a\left((n-j) \ddot{\Lambda}_{0}+j \ddot{\Lambda}_{1}\right) \\
& \quad=\sum_{i=k}^{m+k} \frac{4}{n+2} \sin \frac{(2 i-j+1) \pi}{n+2} \sin \frac{(j+1) \pi}{n+2} \sqrt{\frac{2}{n+2}} \sin \frac{(2 i-j+1) \pi}{n+2} \\
& \quad=\sqrt{2}(n+2)^{-3 / 2} 4 \sin \frac{(j+1) \pi}{n+2} \sum_{i=k}^{m+k} \sin ^{2} \frac{(2 i-j+1) \pi}{n+2}
\end{aligned}
$$




$$
\begin{aligned}
& =\sqrt{2}(n+2)^{-3 / 2} 4\left(\sin \frac{(j+1) \pi}{n+2}\right) \frac{(n+2)}{4} \\
& =\sqrt{\frac{2}{n+2}} \sin \frac{(j+1) \pi}{n+2}=a\left(\Lambda_{j}\right) .
\end{aligned}
$$

Notice that in the first equality the dimensions corresponding to $L\left(\dot{\lambda}_{0}^{\prime}+\dot{\lambda}_{j}\right)$ and $L\left(\dot{\lambda}_{0}+\dot{\lambda}_{j}\right)$ have already been added, which accounts for the coefficient 4 . The third equality is classical, see [K-W], p. 179.

In the case $n=2 m$, the proof that the asymptotic dimensions of both sides coincide is similar.

\section{Branching Rules for $\operatorname{so}(2 m+1) \oplus \operatorname{so}(2 n+1) \subset \operatorname{so}(2(m+n+1))$}

As in $[K$, p. 213], all the decompositions are easily derived by using (1.20) and asymptotics $(0.11),(0.12)$ :

$$
\begin{aligned}
L\left(\Lambda_{0}\right) & =L\left(\dot{\Lambda}_{0}\right) \otimes L\left(\ddot{\Lambda}_{0}\right)+L\left(\dot{\Lambda_{1}}\right) \otimes L\left(\ddot{\Lambda}_{1}\right), \\
L\left(\Lambda_{1}\right) & =L\left(\dot{\Lambda}_{0}\right) \otimes L\left(\ddot{\Lambda}_{1}\right)+L\left(\dot{\Lambda_{1}}\right) \otimes L\left(\ddot{\Lambda}_{0}\right), \\
L\left(\Lambda_{m+n-1}\right) & =L\left(\dot{\Lambda}_{m}\right) \otimes L\left(\ddot{\Lambda}_{n}\right), \\
L\left(\Lambda_{m+n}\right) & =L\left(\dot{\Lambda}_{m}\right) \otimes L\left(\ddot{\Lambda}_{n}\right) .
\end{aligned}
$$

\section{Conclusion}

We list in the following table the infinite families of conformal embeddings together with their index and the references where the corresponding branching rules were computed.

\begin{tabular}{lll}
\hline Embedding & Index & References \\
\hline$s u(m) \times s u(n) \times u_{1} \subset s u(n+m)$ & $(1,1,-)$ & {$[\mathrm{K}-\mathrm{W}]$} \\
$s o(m) \times s o(n) \subset s o(n+m)$ & $(1,1)$ & {$[\mathrm{K}-\mathrm{W}]$, this paper } \\
$s u(n) \times u_{1} \subset \operatorname{so}(2 n)$ & $(1,-)$ & {$[\mathrm{K}-\mathrm{W}]$} \\
$s o(n) \subset s u(n)$ & 2 & {$[\mathrm{~K}-\mathrm{W}]$} \\
$u(n) \subset s p(2 n)$ & 2 & {$[\mathrm{~K}-\mathrm{W}]$} \\
$h \subset \operatorname{sim} h)$ & $\mathrm{h}(h)$ & {$[\mathrm{K}-\mathrm{W}]$} \\
$s u(n) \subset s u(n(n+1) / 2)$ & $\mathrm{n}+2$ & this paper \\
$s u(n) \subset s u(n(n-1) / 2)$ & $\mathrm{n}-2$ & this paper \\
$s u(m) \times s u(n) \subset \operatorname{su}(n m)$ & $(\mathrm{n}, \mathrm{m})$ & {$[\mathrm{A}-\mathrm{B}-\mathrm{I}],[\mathrm{W}]$} \\
$s p(2 m) \times s p(2 n) \subset \operatorname{so}(4 n m)$ & $(\mathrm{n}, \mathrm{m})$ & {$[\mathrm{K}-\mathrm{P}],[\mathrm{V}]$} \\
$s o(m) \times \operatorname{so}(n) \subset \operatorname{so}(n m)$ & $(\mathrm{n}, \mathrm{m})$ & {$[\mathrm{K}-\mathrm{P}],[\mathrm{V}](n m$ even $)$} \\
$s o(n) \times \operatorname{su}(2) \subset \operatorname{sp}(2 n)$ & $(4, \mathrm{n})$ & this paper \\
\hline
\end{tabular}

All the cases when $\bar{g}$ is exceptional were computed in [K-S].

Now it is possible to apply some well known methods to construct modular invariant partition functions. Using the branching rules found in $\& 1$ and $\& 2$, 
we get by restricting a partition function built from the level one characters of $S U(N(N \pm 1) / 2)_{1}$ partition functions for $S U(N)$ of level $N \pm 2$ respectively. Notice that from the classification of level one partition functions for $S U(N)$ we have that in $S U(N(N+1) / 2)$ there are always off-diagonal representatives, since $N(N+1) / 2$ is not prime for $N>2$.

Using the decompositions from Sect. 3, we can restrict a partition function attached to level one characters of $\operatorname{Sp}(N)$ and then contract with a level $N$ partition function of $S U(2)$ and in this way we obtain partition functions for $S O(N)$ of level four.

Acknowledgements. One of us (F.L) would like to thank Prof. V. Kac for his introduction into this subject and into this problem in particular. J.L. thanks W.L. and C.B. for useful discussions.

\section{References}

[A-B-I] Altschuler, D., Bauer, M., Itzykson, C.: Commun. Math. Phys. 132, 349-364 (1990)

[A-G-O] Arcuri, R.C., Gomes, J.F., Olive, D.I.: Nucl. Phys. B 285, 327-339 (1987)

[B-B] Bais, F., Bouwknegt, P.: Nucl. Phys. B 279, 561 (1987)

[B-H] Bardakci, K., Halpern, M.: Phys. Rev. D 3, 2493-2506 (1971)

[G-K-O] Goddard P., Kent, A., Olive, D.: Phys. Lett. B 152, 88-93 (1985)

[H] Halpern, M.: Phys. Rev. D 4, 2398-2401 (1971)

[K] Kac, V.: Infinite dimensional Lie algebras. Third edition. Cambridge: Cambridge University Press, 1990

[K-P] Kac, V., Peterson, D.: Proc. Natl. Acad. Sci. USA 78, No. 6, 3308-3312 (1981)

[K-S] Kac, V., Sanielevici, M.: Phys. Rev. D 37, 2231-2237 (1988)

[K-W] Kac, V., Wakimoto, M.: Adv. in Math. 70, 156-234 (1988)

[N] Nahm, W.: Duke Math. J. 54, 579-613 (1987)

[P] Parthasarathy, R.: Ann. Math. 96, 1-30 (1972)

[S-W] Schellekens, A., Warner, N.: Phys. Rev. D 34, 3092-4010 (1986)

[V] Verstegen, D.: Commun. Math. Phys. 137, 567-586 (1991)

[W] Walton, M.A.: Nucl. Phys. B 322, 775-790 (1989)

Communicated by G. Felder 